\title{
Enzyme kinetics of fungal glucuronoyl esterases on natural lignin-carbohydrate complexes
}

Mosbech, Caroline; Holck, Jesper; Meyer, Anne; Agger, Jane Wittrup

Published in:

Applied Microbiology and Biotechnology

Link to article, DOI:

10.1007/s00253-019-09797-w

Publication date:

2019

Document Version

Other version

Link back to DTU Orbit

Citation (APA):

Mosbech, C., Holck, J., Meyer, A., \& Agger, J. W. (2019). Enzyme kinetics of fungal glucuronoyl esterases on natural lignin-carbohydrate complexes. Applied Microbiology and Biotechnology, 103(10), 4065-4075. https://doi.org/10.1007/s00253-019-09797-w

\section{General rights}

Copyright and moral rights for the publications made accessible in the public portal are retained by the authors and/or other copyright owners and it is a condition of accessing publications that users recognise and abide by the legal requirements associated with these rights.

- Users may download and print one copy of any publication from the public portal for the purpose of private study or research.

- You may not further distribute the material or use it for any profit-making activity or commercial gain

- You may freely distribute the URL identifying the publication in the public portal 
Online Resources

\section{Enzyme Kinetics of Fungal Glucuronoyl Esterases on Natural Lignin-Carbohydrate Complexes}

Applied Microbiology and Biotechnology

Caroline Mosbech, Jesper Holck, Anne Meyer, Jane Wittrup Agger

Protein Chemistry and Enzyme Technology

Department of Biotechnology and Biomedicine, Technical University of Denmark, Søltofts Plads 221, DK-2800

Kgs. Lyngby

Corresponding author:

Jane Wittrup Agger: jaag@dtu.dk

\section{Contents}

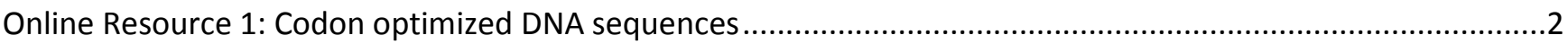

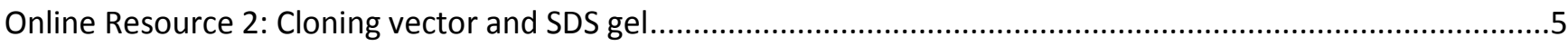

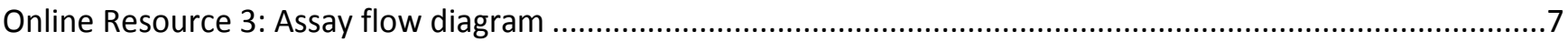

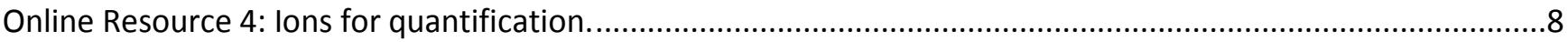

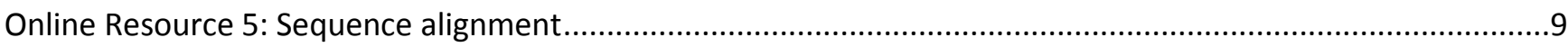

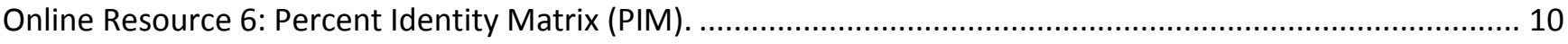

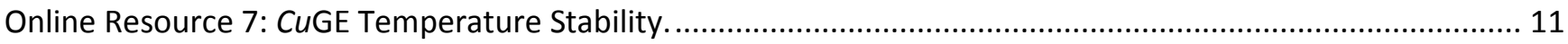




\section{Online Resource 1: Codon optimized DNA sequences}

Armillaria fuscipes, AfGE

>GAATTCGGTTGTTCTGATTTGCCATCTAACATTCCATTGACTCCTAATTCTATGTTGCCAGATCCTTTTACTTTCTTGAAT GGTACTGCTGTTACTTCTTTGTCTGCTTTTACTTGTAGACAAGCTGAAATTTCTGCTTTGTTGCAACAATTCGAG TTGGGTCAATTGCCACCTAGACCTGAATCTGTTGTTGGTACTTTGTCTGGTAACACTTTGACTATTAATGTTGCT CATTCTGGTAGATCTATCTCTTTTACTGTTGGTATTCAAAAGCCAACTGGTTCTGGTACTGGTCCATTTCCTGCT TTGATTGTTTTGGGTGGTTTCTTGTCTTTGCCTCAACCACCTGGTGTTGCTATCTTGTCTTTCAACAACGATGAT ATTGCTGCTCAAAACAATGCTCAATCTAGAGGTCAGGGTAAATTTTTCACTTTGTACGGTTCTAACCACCCAGCT GGTGCTTTGATGGCTTGGGCTTGGGGTGTTGCTAGAATTATGGATGCTTTGGAGCAAGTTAACTCTAACATCGAT TTGACTAGAGTTGGTGTTACTGGTTGTTCTAGAAACGGTAAAGGTGCTTTGGTTGCTGGTGCTTTTGAGCCAAGA ATTGCTTTGACTATTCCTCAAGAATCTGGTTCTGGTGGTGCTGGTTGTTGGAGAATTTCTGATGCTATGTTGGCT TCTGGTGTTTCTACTCAAACTGCTTCTGAAATTGTTCAAGAGAACGTTTGGTTTTCTCCTGCTTTTAATACTTTC GCTCAATCTGGTGTTAACCCATTGCCTTTCGATCATCACATGTTGTCTGCTTTGATTGCTCCAAGACCTTTGTTG GTTATTGATAACACTGGTATCGATTGGTTGGGTCCAGAGTCTGTTTGGGGTTGTCAAAAGACTGCTTCTTTGGTT TGGAAAGCTTTGGGTGTTCCTGATAGAATGGGTGTTTCTCAAGTTGGTAACCATGCTCACTGTGCTTTTCCAACT CAAGAAAAGCCTGATTTGGATGCTTTCGTTAACAGATTCTTGTTGGGTCAAGGTGGTGTTAACACTTCTGGTATT GTTAAGACTGATGGTGCTAATGGTTTGGGTTTCGTTGATTCTAGATGGGTTGGTTGGAAAGTTCCAAACTTGGCT GGTGTCGAC

Thielavia terrestris, TtGE

>GAATTCGCTAGACAAGGTGGTGCTGGTAACGCTACTATTCAATGTGCTGCTATTCCATCTCCTTTTCCAACTTGGCAACAA TTGCCTCAACAATCTACTTTGCCTGATCCATTCTTGCCTTTGAAGTACACTACTCCATCTGATGCTCAAAATATT ATGGCTGGTAAAGGTCAAGGTAGAGTTCAAACTCCTGATGAATGGTATCAATGTAGACAACCAGAAATTTTGCAA ATGTTGCAAGAGTACCAATATGGTTACTATCCTGATCATTCTCAAGAAAAGGTTGAGGCTACTAGATCTGGTACT ACTTTGAACATTGCTGTTACTGCTGGAGATAAGACTGGTTCTTTTAAAGCTTCTTTCACTTTGCCATCTGGTGCT TCTGCTTCTAAACCTGCTCCAGTTGTTATTAACATTGGTGGTATGCAAAATCAACCTTACTTGTCTGCTGGTATC GCTATCGCTCAATTCGATTACACTTCTGTTGCTCCAGATTCTAACTCTAAGACTGGTGCTTTCTGGTCTATCTAC AATGGTAGAGATATTGGTGTTTTGACTGCTTGGGCTTGGGGTTTCCACAGAGTTTTGGATGCTATTAATATGACT GTTCCAGAAATTGATGCTACTAGAGTTGGTGTTACTGGTTGTTCTAGATTGGGTAAAGGTGCTTTGGCTGCTGGT TTGTTCGATAAGAGAATCACTTTGACTATGCCTATGTCTTCTGGTGTTCAGGGTATGGGTCCATACAGATACTAT ACTATGTCTGGTCAAGGTGAAAACTTGGAAAACTCTAAACAAGGTGCTCCTTGGTGGACTGATTCTACTTTGGGT ACTTTTGTTAACCATGCTGAGAATTTGCCTTATGATGCTCACACTATTGCTGCTGCTATTGCTCCAAGAGCTTTG GTTATTGATCAAGGTACTGGAGATCAATTCACTAACTCTAAGGCTACTGCTGTTATTATCTACCCAGCTGCTAAA TTGGTTTATGATTGGTTGGGTGTTGGAGATCAAATTGCTATGTCTGTTAGATCTGGTGGTCATTGTGATATGTCT GGTTACACTTCTGTTTTGCCTTACGTTCAAAAGATTTTCTTTGGTACTCCAACTAACAAAGATTACAACTCTTTG GGTTCTTATGGTTCTCCTATTACTACTGCTTATCCATGGGCTACTGCTACTCCACCTTCTAAGGCTGCT GTCGAC 
Punctularia strigosonata, PsGE

$>$ GAATTCCAATCTACTTGTGATACTCCATCTACTATTGCTGGTTATTCTAACGCTATGTTGCCAGATCCTTTTCAATTCCCT AATGGTACTAGAGTTACTACTGTTGATGAATGGAACTGTAGAAGATCTCAAATTGCTGCTTTGGTTCAAGGTTAC GAGGCTGGTACTTTGCCACCTAAGCCATCTTCTGTTACTGGTTCTTTTTCTGGTAACACTTTGACTGTTAACGTT AACAACGGTGGTTCTTCTATCTCTTTCGCTAACACTATCACTTACCCTTCTGGTACTGCTCCAGCTGGTGGTTGG CCTTTGTTGATTGCTTATGATGGTGGTTCTATTCCAGTTCCTTCTGGTGTTGCTGTTTTGAACTACAACATCGGT AACATCGCTCAACAAACTGATACTACTTCTAGAGGTGTTGGTTTGTTTTACAACTTGTACGGTAAAACTGCTACT GCTTCTGCTATGACTGCTTGGGTTTGGGGTTTGTCTAGAATCATCGATGTTTTGGAAGTTACTCCAGCTGCTATG GTTAACACTGCTAAGTTGGCTGTTACTGGTTGTTCTAGAGATGGTAAAGGTGCTTTGATGGCTGGTGCTTTCGAA CCTAGAATTGCTTTGACTATTGCTCAAGAGTCTGGTGCTGGTGGAGATGCTTGTTGGAGAATTACTAAAACTGAA GGAGATGAGGGTGTTGGTGTTCAACCATCTACTGAAATTGTTCAAGAGAACGTTTGGTTCTCTACTAACTTCAAC AACTACGTTTCTAACTTGAATGTTTTGGCATATGATCATCACTTGTTGGCTGCTATGATTGCTCCTAGAGCTTTG TTGTCTTACGGTAATACTGATTATGCTTGGTTGTCTCCTATGTCTTCTTGGGGTTGTATGAATGCTGCTCATACT GTTTGGCAAGCTTTGGGTGTTCCACAAAACCACGGTTTTGTTGAGGTTGGTGGTCATGCTCACTGTGCTTTCCCT ACTTCTTTGGTTCAAGGTTTGGATGCTTTCTTTGATACTTTCTTGTTGGGTCAAACTGTTTCTACTGATTACTTC TCTACTAACAACGTTTTCGGTTCTGCTCAATGGGTTCCAGCTAACTTCATTAATTGGACTGCTCCTGCTTTGACT GGTTCTTCTACTTCTTCTTCTACTTCTGTTATTTCTTCTATTACTCCAACTCCTGTTCCAACTACTTCTCCTACT ACTGTTCCAACTACTTCTGCTCCTGCTCCAACTGGTGCTCCAGTTGCTAAATGGGGTCAATGTGGTGGTATTGGT TGGACTGGTTCTACTACTTGTACTTCTGGTACTACTTGTACTGTTTTGAACGCTTACTATTCTCAATGTTTG GTCGAC

Cerrena unicolor, CUGE

>GAATTCCAAGCATCCGCTCCTCAATGGGGTCAATGTGGTGGTATCGGTTGGACTGGTCCT ACTGCCTGCCCTAGTGGTTGGGCCTGTCAGCAATTGAACGCTTACTATTCTCAATGTTTG CAAGGTGCTGCTCCTGCTCCAGCTAGAACTACTGCTGCTCCACCTCCACCTCCAGCTACT ACTGCTGCTCCTCCACCTCCAACTACTTCTGCTCCTACTGGTTCTTCTCCAGTTGCTGGT GCTTGTGGTGCTATTGCTTCTACTGTTCCAAACTATAACAATGCTAAGTTGCCTGATCCA TTCACTTTCGCTAATGGTACTGCTTTGAGAACTAAAGCTGATTGGTCTTGTAGAAGAGCT GAAATTTCTGCTTTGATTCAAAACTACGAGGCTGGTACTTTGCCTCCAAAGCCTCCAGTT GTTACTGCTTCTTTTTCTAAATCTGGTAACACTGGTACTTTGGCTATTACTGCTGGTTTG TCTAATTCTCAAACTATTAAATTCTCTCCTACTATCTCTTACCCATCTGGTACTCCTCCA GCTAATGGTTGGCCATTGATTATTGCTTATGAAGGTGGTTCTATTCCTATTCCAGCTGGT GTTGCTACTTTGACTTACTCTAACTCTGATATGGCTCAACAAAATTCTGCTTCTTCCAGA GGTCAAGGTTTGTTTTACCAATTGTATGGTTCTACTCATTCTGCTTCTGCTATGACTGCT TGGGTTTGGGGTGTTTCCAGAATTATTGATGCTTTGGAGATGACTCCTACTGCTCAAATT AATACTCAAAGAATCGGTGTTACTGGTTGTTCCAGAGATGGTAAAGGTGCTTTGATGGCT GGTGCTTTCGAAGAGAGAATTGCTTTGACTATTCCACAAGAATCTGGTTCTGGTGGAGAT GCTTGTTGGAGATTGTCTAAGTACGAGATCGATAACGGTAACCAAGTTCAAGATGCTGTT GAAATTGTTGGAGAGAACGTTTGGTTCTCTACTAACTTCAACAACTACGTTCAAAAGTTG CCTACTGTTCCAGAAGATCATCACTTGTTGGCTGCTATGGTTGCTCCTAGAGCTATGATC TCTTTCGAGAACACTGATTATTTGTGGTTGTCTCCAATGTCTTCTTTCGGTTGTATGACT 
GCTGCTCATACTGTTTGGCAAGGTTTGGGTATTGCTGATTCTCACGGTTTTGCTCAAGTT GGTGGTCATGCTCACTGTGCTTGGCCTTCTTCTTTGACTCCACAATTGAACGCTTTTATT AACAGATTCTTGTTGGATCAATCTGCTACTACTAATGTTTTCACTACTAACAATCAATTC GGAAAGGTTCAGTGGAATGCCGCAAATTGGATTACTTGGACTACCCCTACTCTTACTGGT CTAGA 


\section{Online Resource 2: Cloning vector and SDS gel}

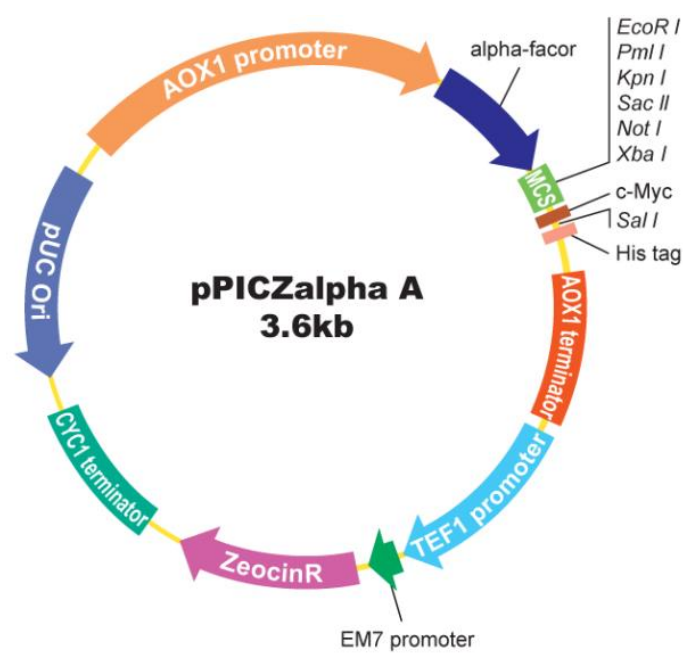

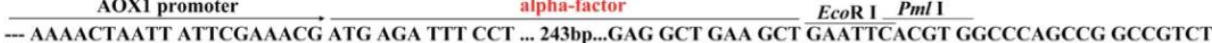
M R F P ...81aa ... E A A E A Kpn I Sac II Not I $X b a \mathbf{I}$ e-mye tag

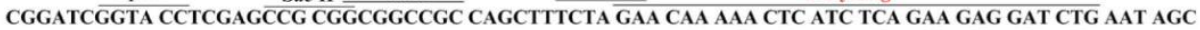
Sal I CCG CGGC

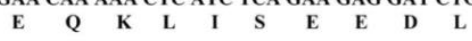

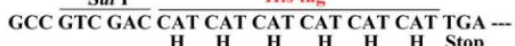

Graphic representation of the $p$ PICZalphaA vector delivered by Genscript holding the CE15 genes between the EcoRI and Sall restriction sites (pPICZalpha-A vector (2019) Genscript https://www.genscript.com/gsfiles/vector-map/yeast/pPICZalphaA.pdf?1897614803 Accessed March $12^{\text {th }}$ 2019) 


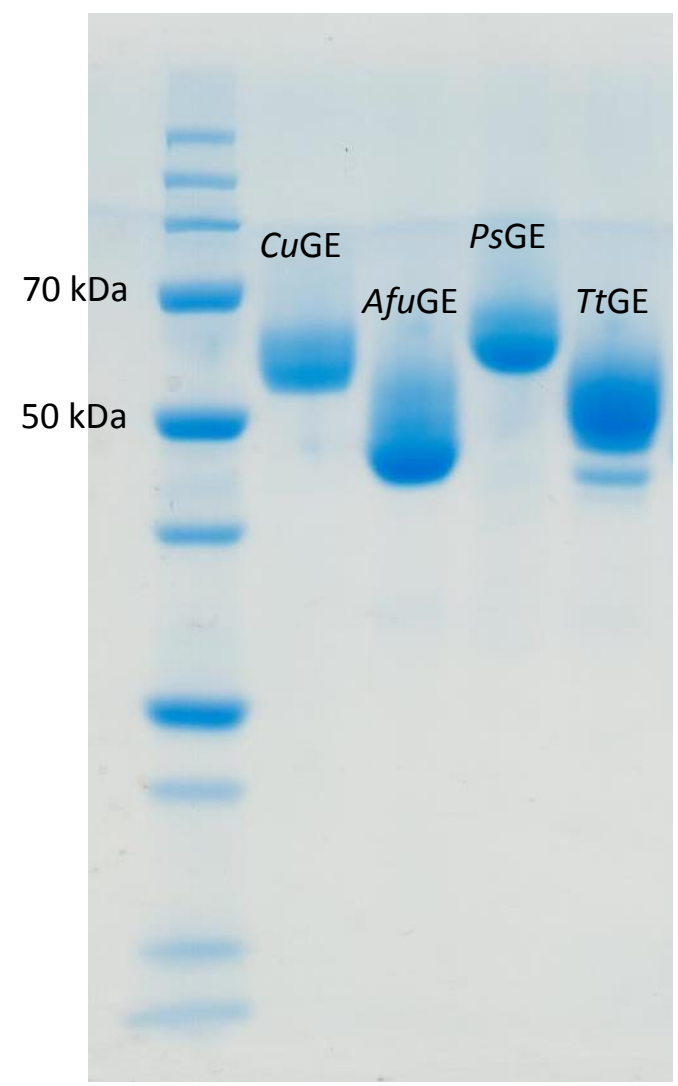

SDS gel of the expressed enzymes after purification. Loaded in the following order with theoretical molecular weight given in bracket; CuGE (48 kDa), AfuGE (40 kDa), PsGE (47 kDa), TtGE (42 kDa). The four enzymes are all glycosylated resulting in the smear and the increase in size compared to the theoretical weight. 


\section{Online Resource 3: Assay flow diagram}

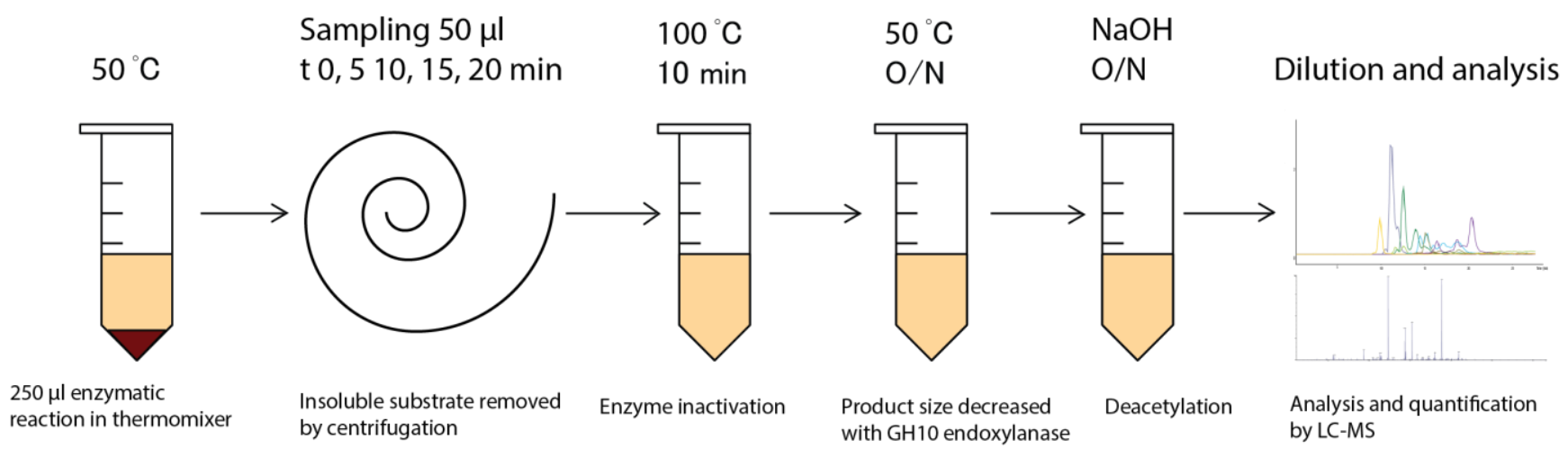

Schematic overview of assay procedure for the kinetic study. Varying substrate concentrations of LRP with a fixed enzyme concentration. Assay conducted in eppendorph tubes in thermomixer. $50 \mu \mathrm{L}$ samples taken every 5 minutes. Reaction stopped after initial removal of substrate by centrifugation and heat inactivation at $100{ }^{\circ} \mathrm{C}$ for $10 \mathrm{~min}$. GH10 endo-xylanase added to the reaction supernatant to decrease the size of the products released by CE15 enzymes. Acetylation removed from the products by alkaline treatment to simplify the products profile. Characterization and quantification of products done by LC-MS. 
Online Resource 4: Ions for quantification.

Complete list of adducts used for quantification of enzyme reaction products and their corresponding retention times.

\begin{tabular}{l|c|c|l}
\hline \multicolumn{1}{c|}{ Analyte } & $m / z[\mathrm{M}+\mathrm{Na}]^{+}$ & $m / z\left[\mathrm{M}+\mathrm{NH}_{4}\right]^{+}$ & $\begin{array}{l}\text { Retention time } \\
(\min ), \text { peak max }\end{array}$ \\
\hline MeGlcAXyl & 363 & 358 & 7.6 \\
\hline MeGlcAXyl $_{2}$ & 495 & 490 & 9.8 \\
\hline MeGlcAXyl $_{3}$ Reduced MeGlcAXyl $_{3}$ & 627 & 622 & 12.6 \\
\hline
\end{tabular}




\section{Online Resource 5: Sequence alignment}

Alignment of segments of the amino acid sequences from CE15 characterized in this study. Conserved GCSRXG motif with a catalytic nucleophile serine marked with red curly bracket. Catalytic glutamic acid and histidine marked with red arrow. TtGE belonging to PPR group 8 holds a serine at the position of the glutamic acid.

Aligment and sequence logo made with CLC Workbench 7.5.

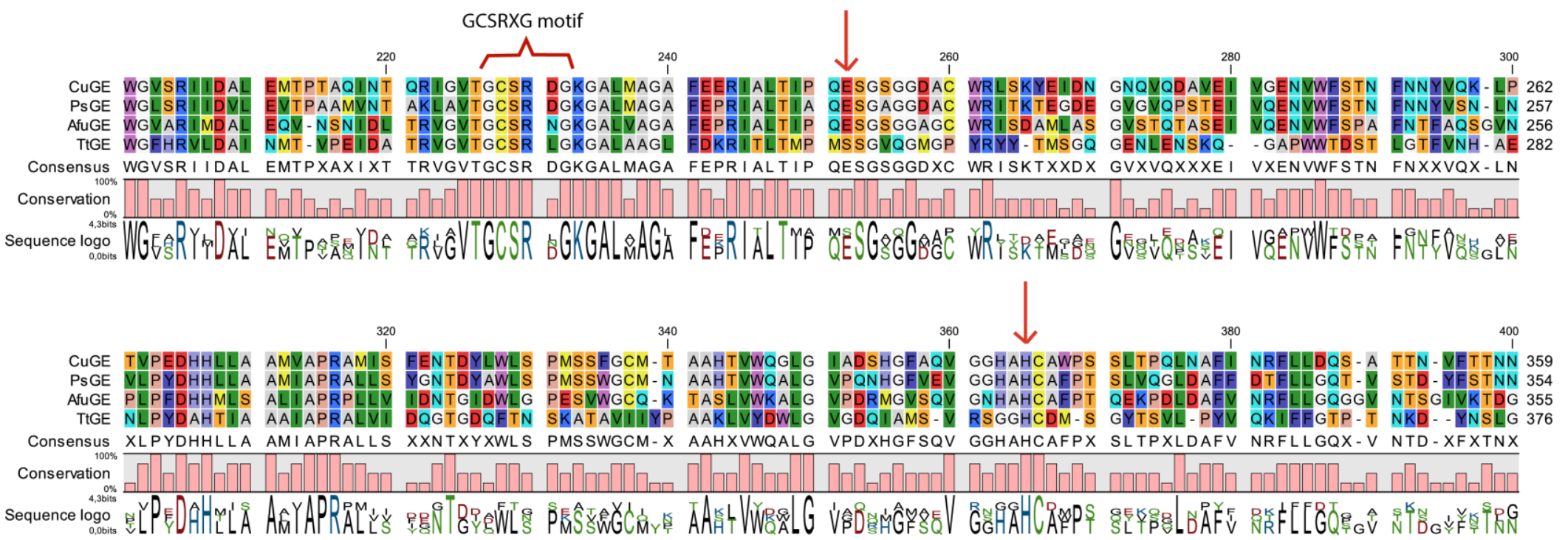




\section{Online Resource 6: Percent Identity Matrix (PIM).}

Percent query coverage given in brackets. Color scale from light to dark orange indicate percent sequence identity from the lowest value $28 \%$ in light orange to the highest value $100 \%$ in dark orange. Values obtained using BLAST (https://blast.ncbi.nlm.nih.gov).

\begin{tabular}{|l|r|r|r|r|}
\hline & \multicolumn{1}{|l|}{ TtGE } & \multicolumn{1}{l|}{ AfuGE } & \multicolumn{1}{l|}{ PsGE } & \multicolumn{1}{l|}{ CuGE } \\
\hline TtGE & $100(100)$ & $35(84)$ & $31(79)$ & $28(96)$ \\
\hline AfuGE & $35(84)$ & $100(100)$ & $51(99)$ & $49(99)$ \\
\hline PsGE & $31(77)$ & $51(89)$ & $100(100)$ & $65(88)$ \\
\hline CuGE & $28(98)$ & $49(99)$ & $65(97)$ & $100(100)$ \\
\hline
\end{tabular}




\section{Online Resource 7: CuGE Temperature Stability.}
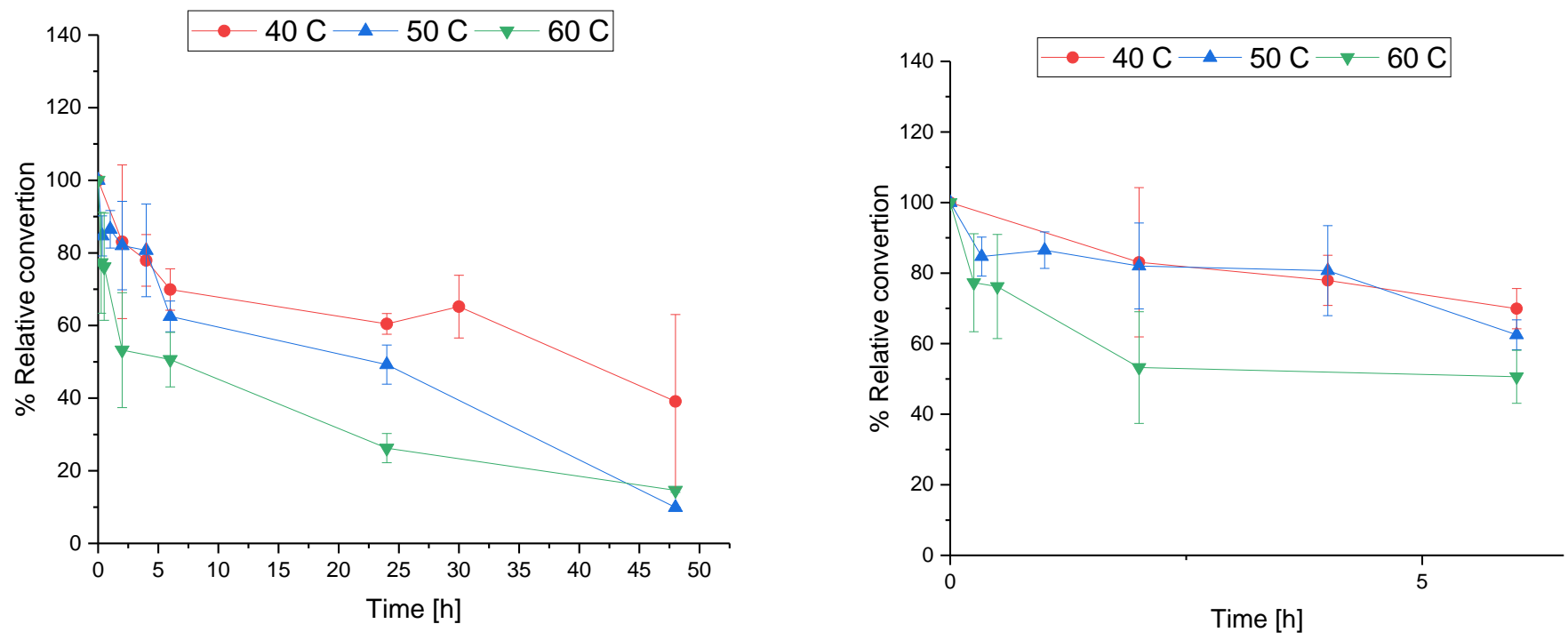

Thermostability of CUGE obtained by incubation at specific temperatures for 48 hours and followed by activity measurement on Me-MeGlcA (left graph). \% conversion calculated relative to activity with CuGE not preexposed to incubation. Rigth graph: A zoom of the first 6 hours of incubation. The study was conducted in triplicates. The kinetic assays (Fig 2) were conducted for $20 \mathrm{~min}$ at $50{ }^{\circ} \mathrm{C}$. Under these conditions CuGE still retained around $85 \%$ of its relative activity. After 25 hours at $50{ }^{\circ} \mathrm{C}$ approx. 50\% relative activity remained. 